\title{
Flow cytometric detection of circulating dendritic cells in healthy subjects
}

\author{
B. Rovati, S. Mariucci, M. Manzoni, K. Bencardino, M. Danova \\ Flow Cytometry and Cellular Therapy Unit, Medical Oncology, IRCCS Foundation San Matteo University \\ Hospital, Pavia, Italy
}

ii

(C)2008 European Journal of Histochemistry

Dendritic cells (DCs) are the key antigen-presenting cells controlling the initiation of the $T$ cell- dependent immune response. Currently, two peripheral blood DC subsets have been identified on the basis of their CD11c expression. The CD11c-negative (CD11C-) DCs (expressing high levels of CD123) are designated as lymphoid-derived DCs (DC2), whereas the $\mathrm{CD} 11 \mathrm{C}^{+} / \mathrm{CD} 123^{-}$cells, do identify the myeloidderived DCs (DC1). A growing number of studies have been conducted in recent years on both the quantitative and functional alterations of DCs and their subsets in different pathological conditions. In the present study we assessed, using two different flow cytometric (FCM) techniques, the normal profile of blood DCs in 50 italian adult healthy subjects (M/F: $25 / 25$, median age 42.5 years, range $20-65)$. The percentage and the absolute number of DCs and their subsets, were obtained starting from whole blood samples in two ways: 1) by calculating the number of DCs when gated as lineage-negative/ $\mathrm{HLA}^{-\mathrm{DR}^{+}}$and identifing the two subsets as CD11 ${ }^{+}$ (DC1) and $\mathrm{CD}_{123^{+}}$(DC2) and 2) by using three specific markers: BDCA.1 (CD11 $\mathrm{c}^{+}$high/CD123+ low, myeloid DCs); BDCA.2 (CD11C-/ CD123+high, lymphoid DCs); BDCA.3 (CD11 $\mathrm{C}^{+}$low /CD123-, myeloid DCs). Six parameters, 4-color FCM analysis were perfomed with a BD FACSCanto equipment. The mean values of the percentage and of the absolute number were: $0.5 \pm 0.2 \%$ and $30 \pm 11$ cells/ $\mu \mathrm{L}$ for $\mathrm{DCs}$; $0.2 \pm 0.1 \%$ and $15 \pm 6$ cells/ $\mu \mathrm{L}$ for $\mathrm{DC} 1 ; 0.2 \pm 0.1 \%$ and $15 \pm 7$ cells/ $\mu \mathrm{L}$ for DC2. The same values were: $0.2 \pm 0.1 \%$ and $16 \pm 7$ cells $/ \mu \mathrm{L}$ for BDCA.1; $0.2 \pm 0.1 \%$ and $12 \pm 7$ cells $/ \mu \mathrm{L}$ for BDCA.2; $0.02 \pm 0.01 \%$ and $2 \pm 1$ cells/ $\mu \mathrm{L}$ for BDCA.3, respectively. Our study confirmes that the two types of FCM analysis are able to identify the DC population. We also provides the first reference values on normal rates and counts of blood DCs in italian adult healthy subjects.

Key words: Circulating dendritic cells, flow Cytometry, normal reference values.

Correspondence: Bianca Rovati,

Flow Cytometry and Cell Therapy Unit Medical Oncology

IRCCS Foundation S. Matteo University Hospital

27100 Pavia, Italy

Tel.:+39.0382.502523

Fax:+39.0382.528434

E-mail: flow.cytometry@smatteo.pv.it

Paper accepted on February 4, 2008

European Journal of Histochemistry

2008; vol. 52 issue 1 (Jan-Mar): 45-52
$\mathrm{D}$ endritic cells (DCs), are specialized and co-stimulatory cells which play an important role in the induction of the cellular immune response (Hart, 1997). These cells can be found in many tissues of the human body, as an heterogeneous population of specialized presenting cells, circulating via the blood to most of the tissue (Steinmann, 1973). Many studies revealed that DCs play a pivotal role in the induction of $T$ cell responses resulting in cell-mediated immunity and that they are also able to initiate and regulate $\mathrm{T}$ and B- lymphocyte responses, to activate these cells, and to tolerize $\mathrm{T}$ cells to self-antigens $(\mathrm{Ho}$, 2001; Lukacs-Kornek, 2008; Shurin, 2007; Sozzani, 2007; Thurnher, 2007; Wilczynski, 2008).

Two peripheral blood DC subsets (DC1 and DC2) have been described (Liu, 2001, a; MacDonald, 2002). The CDllc-negative (CDIlc) DCs, which usually express high levels of CD123 $\left(\mathrm{CD}_{12} 3^{+}\right)$, are designated lymphoid-derived DCs, whereas the CDIlc-positive $\left(C D 1 l C^{+}\right)$DC subset generally expresses low levels of CD123 (CD123-) and represents the myeloid-derived DCs (Shortman, 2002). Both subsets express high level of HLA-DR $\left(\mathrm{HLA}-\mathrm{DR}^{+}\right)$and lack the lineage markers CD3, CD14, CD16, CD19, CD20 and CD56, several functional differences between $\mathrm{CDI} 1 \mathrm{C}^{+} / \mathrm{CD} 123^{-}$and $\mathrm{CDIIC}^{-} / \mathrm{CDI}_{23^{+}} \mathrm{DCs}$ have been described (Ardavin, 2001). While both the DC1 and DC2 subset induce strong proliferation of naïve CD4 $4^{+}$cells, upon interaction with $\mathrm{T}$ cells, the $\mathrm{DCl}$ subset predominantly prime a $T$ helper type 1 (Thl) cell antimicrobial response, the DC2 subset seems to support the generation of a $T$ helper type 2 cell (Th2) response (Rissoan, 1999; Grouard, 1997).

Flow cytometric analysis of circulating DCs and their subsets has proven a useful tool in both experimental and clinical studies on several pathological conditions such as: autoimmunity, immunodeficien$c y$, infections, transplantation and, more recently, haematological and solid tumors. 
Recently, we have demonstrated that high-dose cyclophosphamide followed by G-CSF (utilized as a mobilization regimen for $\mathrm{CD} 34^{+}$cells into the $\mathrm{PB}$ for autologous transplantation procedures) induces a reversed DC1/DC2 ratio without a significant increase of blood DCs (Ferrari, 2003). We also did not observe any significant changes in the circulating DC pool in advanced breast cancer patients after different schedules of standard-dose chemotherapy as well as during immunotherapy with trastuzumab in $\mathrm{HER}^{+}$-patient subset. We have also shown that both DCs and their subset distribution, are comparable in both early stage and advanced breast cancer patients before starting CT, while, in this latter group, we observed a significant decrease of the DCl subset after the completion of the CT program (Ferrari, 2005). In this field, an important issue for future studies comes from the fact that, due to both the different of potential markers of DCs and the technological advances in FCM equipments, there is not any universally accepted standard method to determine their number and immunophenotype; furthermore, there is still a limited knowledge of the number and distribution of blood DCs in the healthy populations (Narbutt, 2004).

The aims of the present study are: 1) to compare two FCM approaches for the analysis of blood $D C s$, and 2) to provide reference data on normal rates and counts of these cells in a representative italian healthy population.

\section{Materials and Methods}

\section{Study population}

A total of 50 adult healthy subjects ( $25 \mathrm{M}, 25$ F), with a median age of 42.5 years (range: 20-65 years), were examined. Each volunteer gave written informed consent before entry into the study and the experimental trial was conducted after the approval of our local Ethical Committee. The characteristics of the study population are showed in Table 1.

Selection criteria were based on a prospective written survey to disqualify donors associated with a range of known risk categories and on a retrospective serological testing to exclude donors with prior exposure to a range of known patogens. The principal selection criteria of the study population are listed in Table 2.

\section{Monoclonal antibodies (MoAbs) for DCs analysis}

The following mouse anti-human MoAbs directly conjugated were used: fluorescein isothiocyanate (FITC)-labelled anti-CD3 (SK7), -CD16 (B73.1), -CD19 (SJ25C1), -CD20 (L27); -CD14 (M5E2), -CD34 (8G12), -CD56 (NCAM 16.2), -HLA-DR (L243) (BD Bioscences, San Jose, California, USA) and -CDIlb (Bear 1, Coulter Immunotech); allophycocyanin (APC)-labelled anti-HLA-DR, (BD Bioscences); phycoerthrin (PE)-labelled anti-CD19 (SJ25C1), anti-CD11C, -CD123 (BD Bioscences); peridinim chlorophyll (PerCP)-labelled anti-CD45 (2D1) (BD Bioscences); FITC-labelled anti CDIC (BDCA.1, AD58E7), -BDCA.2 (AC144) and PElabelled anti-BDCA.3 (AD5-14H12), (Miltenyi Biotec $\mathrm{GmbH}$, Bergisch Gladbach, Germany), (Table $3 a$ and Table 3b).

\section{Multi-color staining and flow cytometric analysis for DCs}

Dendritic cells are known to be detected as $\mathrm{MCH}$ class II-expressing cells ( $\left.\mathrm{HLA}-\mathrm{DR}^{+}\right)$and lacking of T cell (CD3,CD11b), B cell (CD19, CD20), NK (CD16, CD56), macrophage/monocyte (CD14), and hematopoietic progenitor cell (CD34) markers, so they were defined as lineage-negative (lin-neg)

Table 1. Characteristics of the studied population.

\begin{tabular}{lc}
\hline Number of studies healthy subjects & 50 \\
Gender (F/M) & $25 / 25$ \\
Median age/range (years) & $42,5 / 20-65$ \\
Median number of WBC (cells/ $\mu \mathrm{L})$ & 6447 \\
Median number of lymphocytes (cells/ $\mu \mathrm{L})$ & 2236 \\
\hline
\end{tabular}

Table 2. Summary of main selection criteria of the studied population.

Minimun body weight of $50 \mathrm{Kg}$

Human Immunodeficiency Virus- and Hepatitis B, C - negative

Not pregnant for 12 months prior to blood donation

No medical or dental treatment for 72 hours prior to blood donation

No fever $>38^{\circ} \mathrm{C}$ for 4 weeks $h$ prior to blood donation

No use of medication for 4 weeks prior to blood donation

No needle-stick injury, acupuncture, tattooing, body-piercing or for 12 months prior to blood donation No blood transfusion for 12 months $h$ prior to blood donation

No history of high blood pressure, heart attack, shortness of breath, stroke, or unconsciousness No history of atopic disorders

No family member with diagnosed Creutzfeldt-Jacobs disease

No residence in the UK or $>6$ months during the period 1980-1986

No travel to malaria endemic regions for 6 months prior to blood donation

№ history of Malaria, Leishmanias, Borreliosis, Brucellosis or Toxoplasmosis

No contact with persons exhibiting symptoms of infectious disease for 4 weeks prior to blood donation 
cells. We identified dendritic cell subsets as lin-neg $/ C D 11 C^{+}(D C 1)$ and lin-neg/CD123+ (DC2) or using three specific markers: BDCA.1 (CDllc-high/ CD123-low myeloid DCs in the blood); BDCA.2 (CD1lc-neg/CD123-high lymphoid blood DCs) and BDCA.3 (CDIlc-low/CD123- myeloid DCs) (Dzionek, 2000; Demedts, 2005; Patterson, 2005; Pilichowska, 2007; Tsoumakidou, 2006; Zaba, 2007; Pinzon-Charry, 2005).

For both the techniques, peripheral blood was collected into a $4 \mathrm{~mL}$ Vacutainer (Becton Dickinson, Basel, Switzerland) tube containing liquid tripotassium ethylene diamine tetra-acetic acid ( $\mathrm{K}_{3} \mathrm{EDTA}$ ) as an anticoagulant and processed within $4 \mathrm{~h}$ of collection. All the procedures utilized in this study are conformed to the Helsinki Declaration of 1975 (Anonymous, 2002). Anticoagulated venous whole blood was aliquoted in $100 \mu \mathrm{L}$ amounts into $12 \times 75 \mathrm{~mm}$ polypropilene tubes (Becton Dickinson Labware, Franklin Lake, NJ, USA) and after gently mixed, incubated with the appropriate fluorochrome-conjugated MoAbs at the manifacture recommended concentration for

Table 3a. Monoclonal antibodies employed for the lineage-negative analytical procedure.

\begin{tabular}{|c|c|c|c|c|c|}
\hline Antigen & Fluorochrome & Clone & Isotype & Vendor & Volume $(\mu \mathrm{L})$ \\
\hline CD3 & FITC & SK7 & $\operatorname{lgG1}$ & BD Biosciences & 20 \\
\hline CD11b & FITC & Bear 1 & $\lg M$ & Coulter Immunotech & 10 \\
\hline CD11c & PE & B-ly6 & $\lg G 1$ & BD Biosciences & 20 \\
\hline CD14 & FITC & M5E2 & $\lg G 2 a$ & BD Biosciences & 20 \\
\hline CD16 & FITC & B73.1 & $\lg G 1$ & BD Biosciences & 20 \\
\hline CD19 & FITC & SJ25C1 & $\lg G 1$ & BD Biosciences & 20 \\
\hline CD20 & FITC & L27 & $\operatorname{lgG1}$ & BD Biosciences & 20 \\
\hline CD34 & FITC & $8 \mathrm{G} 12$ & $\lg G 1$ & BD Biosciences & 20 \\
\hline CD45 & PerCP & $2 \mathrm{D} 1$ & $\lg G 1$ & BD Biosciences & 20 \\
\hline CD56 & FITC & NCAM 16.2 & $\lg G 2 b$ & BD Biosciences & 20 \\
\hline CD123 & PE & $9 F 5$ & $\lg G 1$ & BD Biosciences & 20 \\
\hline HLA-DR & APC & L243 & $\lg G 2 a$ & BD Biosciences & 5 \\
\hline
\end{tabular}

Table 3b. Monoclonal antibodies employed for the analytical approach based on specific markers.

\begin{tabular}{lccccc}
\hline Antigen & Fluorochrome & Clone & Isotype & Vendor & Volume $(\mu L)$ \\
\hline CD19 & PE & SJ25C1 & IgG1 & BD Biosciences & 20 \\
CD45 & PerCP & $2 D 1$ & IgG1 & BD Biosciences & 20 \\
CD123 & PE & 9 F5 & IgG1 & BD Biosciences & 20 \\
HLA-DR & FITC & L243 & IgG2a & BD Biosciences & 20 \\
HLA-DR & APC & L243 & IgG2a & BD Biosciences & 5 \\
BDCA.1 & FITC & AD58E7 & IgG2a & Myltenyi Biotec & 5 \\
BDCA.2 & FITC & AC144 & IgG1 & Myltenyi Biotec & 5 \\
BDCA.3 & PE & AD5-14H12 & IgG1 & Myltenyi Biotec & 5 \\
\hline
\end{tabular}

15 min at room temperature (RT) in the dark. Stained whole blood samples were subjected to red blood cells lysis with $2 \mathrm{~mL}$ lysing solution (Auto Lyse BD Biosource, Europe SA.) vortexed and incubated for $15 \mathrm{~min}$ at RT in the dark. Preparated samples were stored at RT in the dark and analysed within $1 \mathrm{~h}$.

Evaluation of nucleated cells from whole blood specimens was performed using a BD FACSCanto flow cytometer (BD Biosciences, San Jose, CA, USA) instrument, with identical set up parameters between samples. Flow cytometry data was analysed using BD FACSDiva software.

Cells labelled with PE-, FITC-, Per-CP- and APC-conjugated isotype MoAbs that were nonreactive to human cells were used as a control to determine the fluorescence background.

A range of internal quality assurance procedures were employed, including daily calibration of the optical alignment and fluidic stability of the instrumentation (by using 7-color SetUp Beads, BD Biosciences). The sensitivity and the stability of cell count was tested using international quality controls purchased from United Kingdom National External Quality Assessment Scheme (UK NEQAS LI, Sheffield, UK) (Whitby, 2002) and daily monitoring of whole-blood preparation procedures and MoAbs reactivity using Immuno-Trol (Beckman Coulter, Fullerton, CA, USA) control cells.

\section{Statistical analysis}

Statistical analysis was performed using Microsoft Excel software. The mean \pm standard deviation of the percentage and of the absolute number of DCs and their subsets were calculated, togheter with their range distribution. Student's ttest was used to compare the DC population identified by the two described FCM approaches. Correlations were considered significant with $p$ value $<0.05$

\section{Results}

\section{Gating strategy for DCs assay}

Gating strategies and whole blood sample analysis were as follow: the threshold was set on PerCP fluorescence ( $F L 3)$ to reduce debris; a gate for leukocyte analysis region was defined in a dot plot of CD45 versus side scatter (SSC). Dendritic cell subpopulations were easily distinguished from other cells. For rare events analysis of DC, at least 
100000 cellular events were acquired from $0.1 \mathrm{~mL}$ of whole blood.

These events were displayed in a lin-neg-FITC versus HLA-DR-APC dot plot in which a second gate was created to identify lineage-negative cells (DCs). In the lineage-negative procedure $\mathrm{DCl}$ and $\mathrm{DC} 2$ subsets were identified as lin-neg / $\mathrm{HLA}^{-\mathrm{DR}^{+} /}$ $\mathrm{CDIIC}^{+}$and lin-neg / HLA-DR $/ \mathrm{CD} 123^{+}$, respectively, after gating on lineage-negative cells. The gating strategy utilized for definition of CDIlc or CD123 on lin-neg/HLA-DR ${ }^{+}$cells was determined as shown in Figure 1 (panel a).

In the second approach based on specific markers, the DC subsets were identified directly from three specific markers: BDCA.1-FITC, BDCA.2FITC and BDCA.3-PE. The gating strategy utilized for definition of BDCA subsets was determined as shown in Figure 2 (panel a).

\section{Dendritic cells and DC subsets identified by the lineage-negative procedure}

The absolute DC number was derived from the total white blood cells count as calculated on an haematological analyser (Coulter, Miami, USA) and the percentage of DC subsets as determined by FCM. a

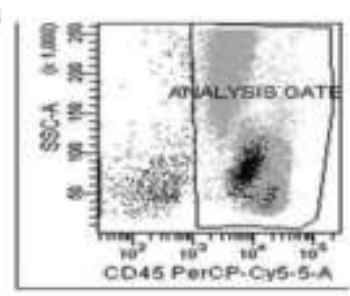

c

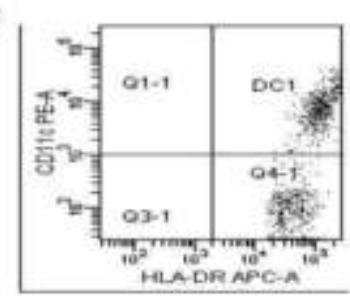

b

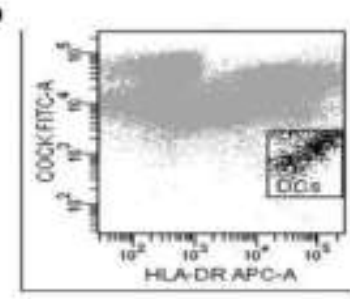

d

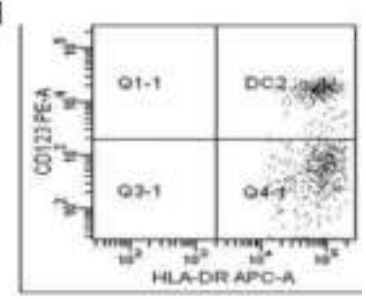

Figure 1. Flow cytometric dot plot panels show representative data relative to the method utilized for the identification of circulating dendritic cells (DC) and DC subsets, with the lineage-negative analytical procedure. Panel a shows leukocyte analysis region applied to PerCP- labelled anti-CD45 versus side scatter (SS) data acquired for exsclusion of debris. Panel b shows the same identified cell population after labelling with a cocktail of FITC-conjugated MoAbs recognizing the lin-neg associated antigens (see Table 3a) and APC-labelled anti-HLA-DR MoAb. Panels $c$ and $d$ show the DC subsets identified from HLA-DR-APC and CD11c-PE for DC1 and HLA-DR-APC and CD123-PE for DC2.
Dot plot panels utilized for definition of CDIlc or CD123 on lin-neg/HLA-DR ${ }^{+}$cells are shown in Figure 1 (panels $b, c, d$ ).

In our healthy population, the mean values of the percentage and of the absolute number were: $0.5 \pm 0.2 \%$ and $30 \pm 11$ cells/ $\mu \mathrm{L}$ for DCs; $0.2 \pm$ $0.1 \%$ and $15 \pm 6$ cells $/ \mu \mathrm{L}$ for $\mathrm{DC} 1 ; 0.2 \pm 0.1 \%$ and $15 \pm 7$ cells/ $\mathrm{LL}$ for $\mathrm{DC} 2$. We also calculated the $D C 1 / D C 2$ ratio obtaining as mean value of the percentage $1.1 \pm 0.5$ (Table 4).

\section{Dendritic cell subsets identified by specific markers}

The expression of BDCA.1, BDCA.2 and BDCA.3 subsets was determined, as shown in Figure 2 (panels $b, c, d$ ). The mean values of the percentage and of the absolute number were: $0.2 \pm 0.1 \%$ and $16 \pm 7$ cells/ $\mu \mathrm{L}$ for BDCA. $1 ; 0.2 \pm 0.1 \%$ and $12 \pm 7 \mathrm{cells} / \mu \mathrm{L}$ for BDCA.2; $0.02 \pm 0.01 \%$ and $2 \pm 1$ cells/ $\mu \mathrm{L}$ for BDCA.3 (Table 4b).

\section{Comparison between DC populations identified by the two different approaches}

No statistically significant difference was found between the sum of the DC1+DC2 cell populations versus the sum of the BDCA.1+BDCA.2+BDCA.3 cell populations. a

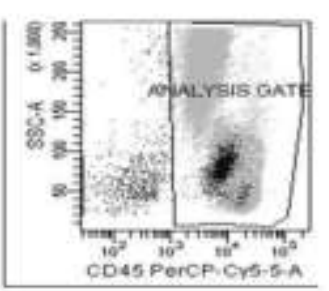

c

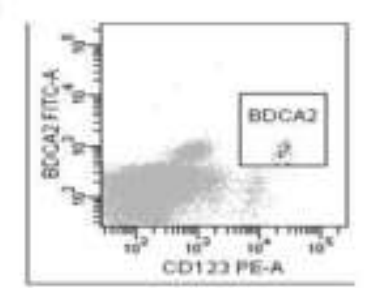

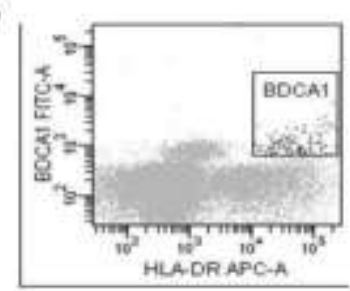

d

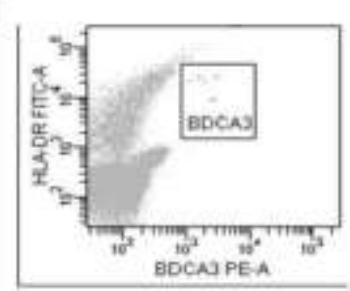

$\overline{\text { Figure 2. Flow cytometric dot plot panels showing representative }}$ data relative to the analytical method for identification of DC subsets by specific markers starting from peripheral blood nucleated cells. Panel a shows the leukocyte analysis region applied to PerCP- labelled anti-CD45 versus side scatter (SS) data acquired for exsclusion of debris. Panel b shows BDCA.1 (CD11chigh/CD123-low myeloid DCs) expression. Panel $c$ and Panel d show BDCA.2 (CD11c-neg/CD123-high lymphoid blood DCs) and BDCA.3 (CD11c-low/CD123 neg myeloid DCs) expression, respectively. 
Table 4a. Mean values of percentage and absolute number of dendritic cells (DCs), DC subsets and their ratio (DC1/DC2) in healthy subjects.

\begin{tabular}{cccc}
\hline DCs & $D C 1$ & $D C 2$ & $D C 1 / D C 2$ (ratio) \\
\hline $0.5 \pm 0.2 \%$ & $0.2 \pm 0.1 \%$ & $0.2 \pm 0.1 \%$ & $1.1 \pm 0.5 \%$ \\
$30 \pm 11$ cells $/ \mu \mathrm{L}$ & $15 \pm 6$ cells $/ \mu \mathrm{L}$ & $15 \pm 7$ cells $/ \mu \mathrm{L}$ & - \\
\hline
\end{tabular}

Table 4b. Mean values of percentage and absolute number of DC subsets (identified by BDCA markers) in healthy subjects.

\begin{tabular}{ccc}
\hline BDCA.1 & BDCA.2 & BDCA.3 \\
\hline $0.2 \pm 0.1 \%$ & $0.2 \pm 0.1 \%$ & $0.002 \pm 0.002 \%$ \\
$16 \pm 7$ cells $/ \mu \mathrm{L}$ & $13 \pm 7$ cells $/ \mu \mathrm{L}$ & $2 \pm 1$ cells $/ \mu \mathrm{L}$ \\
\hline
\end{tabular}

The sum of the mean values of the BDCA.I+ BDCA.2+BDCA.3-positive cells ranged from 0.2 to $0.62 \%$ and, in absolute numbers from 16 to 46 cells/ $\mu \mathrm{L}$, while the sum of the DC1 + DC2 cell populations ranged from 0.2 to $0.61 \%$ and from 17 to $43 \mathrm{cell} / \mu \mathrm{L}$.

The corresponding mean values of the percentage and of the absolute number were $0.42 \pm 0.22 \%$ and $31 \pm 15$ cells/ $\mu \mathrm{L}$ for the BDCA.1+BDCA.2+ BDCA.3, identified subsets. The same values were $0.40 \pm 0.20 \%$ and $30 \pm 13$ cells $/ \mu \mathrm{L}$ for the $\mathrm{DCl}+$ DC2 subsets.

\section{Discussion}

The importance of DCs in the initiation and control of innate and adaptative immune response is well documented. Dendritic cells are specialized for uptake, processing and presentation of antigens to $T$ cells, thus activating and modulating key immune responses. Even if the continuous replenishement of any DC pool by blood monocytes (which are established circulating precursors for DCs) in the steady state remains to be established (Tacke, 2006), it is well known that, in the peripheral blood, DCs cells are present in the immature state and have the capability to internalize different protein antigens through various endocytic mechanisms and subsequently, subject them to endosomal processing. (Banchereau, 2000; Liu, 2001, b; Thurnher, 2007). Upon activation in the presence of inflammatory mediators, DCs undergo to a process of maturation and acquire the capacity to migrate into secondary draining lymph nodes and tissues, where they become efficient in presenting antigens to naïve $T$ lymphocytes. This process is associated with the upregulation of co-stimolatory molecules and the secretion of cytokines that polarize the T cell-mediate immunity to a Th1 or Th2 response (Jung, 1993).

In recent years, there has been an increased interest in the study of peripheral blood DCs and their role (still not completely clarified) in the dynamics of T-cell priming that could be utilized also for practical approaches, for example in vaccine design, cancer therapy and treatment of inflammatory diseases (Henrickson, 2007).

These studies have been facilitated by important technical improvements in the blood DC enumeration methods available (Vuckovic, 2004; Servet, 2002).

By using FCM-based techniques, circulating dendritic cells were first shown to be decreased in HIVinfection (Grassi, 1999; Donaghy, 2001; Finke, 2004), and more recently, in other viral infectious diseases (Pichyangkul, 2003), whereas increased DC counts were found in acute influenza infection (Coates, 2003). Functional and quantitative variations were also described as a function of age, in surgical and physical stress $(\mathrm{Ho}, 2001)$ as well as in advanced breast cancer (Ferrari, 2003; Ferrari, 2005), in B-lineage acute lymphoblastic leukaemia and in other oncological conditions (Mami, 2004; Della Porta, 2005).

In our experience, the ability to enumerate DCs and their subsets, togheter with the study of their function, directly in the PB mononucleated cells of cancer patients, has provided broader understanding of the role of these cells in tumor immunology as well as of their potential applications in the clinical setting (Ferrari, 2005).

No single specific marker, that could be used to exactly identify all the DCs was available until recently. Therefore, a combination of MoAbs, the so called lineage cocktail has been used to easilty identify lin-neg/HLA-DR+ DCs by FCM in PB.

Lin-neg/HLA-DR+ DCs account for 0.1 to $2 \%$ of the peripheral blood nucleated cells in healthy individuals and most of these cells express either CDIlc or CD123 antigens. A panel of MoAbs that identify three presumably novel blood DC antigens has been recently, tested: BDCA.1, which identifies CDllc-high/CD123-low myeloid DCs; BDCA.2 for CDllc-neg/CD123-high lymphoid DCs and BDCA.3 for CD11c-low/CD123-neg myeloid DCs). 
As recently performed on patients with breast cancer (Ferrari, 2005) and on patients affected by coeliac disease (Ciccocioppo, 2007), our present study on healthy subjects was performed using multicolor FCM after staining the whole PB with: 1) a cocktail of lineage-specific MoAbs and 2) with the above mentioned new specific MoAbs.

It is known that BDCA.1 (also known as CDIC)positive cells show CDllc-high and CDI23-low expression, that BDCA.2-positive cells show CD123-high expression and that BDCA.3-positive cells show CDIlc-low expression. With this expression profile, each one of the BDCA markers $(1,2$ or 3) clearly do not identify exactly and exclusively $\mathrm{DC} 1$ or DC2 cell populations, because they react with only one part of the DC1 or of the DC2 cell populations.

Taking into account the above mentioned point, we confirmed that the two analytical methods are able to identify the DC population. Both the FCM approaches we utilized are rapid and sensitive. They allow the determination of circulating DCs and their subsets in a relatively small blood volume so that they can be used in the clinical setting as an additional indicator of the patients' immunocompetence in different pathological conditions.

We also provided the first data about the normal values of blood DCs in adult healthy italian population. This may be helpful in the early detection of underlying immune alterations and it is crucial for creating a range of reference value for comparative clinical studies.

\section{Acknowledgments}

We would like to thank all the volunteers for taking part in the study.

The present work was supported by an Hospital research grant (to M. Danova) from the IRCCS Fondazione S. Matteo, Pavia.

\section{References}

Anonymous. World Medical Association Declaration of Helsinki: ethical principles for medical research involving human subjects. J Postgrad Med 2002;48:206-8.

Ardavin C, Martinez del Hoyo G, Martin P, Anjuere F, Arias CF. Marin $A R$, et al. Origin and differentiation of dendritic cells. Trends Immunol 2001;22:691-700.

Banchereau J, Briere F, Caux C, Davoust J, Lebecque S, Liu YJ, et al. Immunobiology of dendritic cells. Annu Rev Immunol 2000; 18:767-11.

Ciccocioppo R, Ricci G, Rovati B, Pesce I, Mazzocchi S, Piancatelli D et al. Reduced number and function of peripheral dendritic cells in coeliac disease. Clin Exp Immunol 2007;149:487-96.
Coates PT, Barratt-Boyes SM, Zhang L, Donnenberg VS, O'Connell PJ, Logar AJ, et al. Dendritic cell subsets in blood and lymphoid tissue of rhesus monkeys and their mobilization with Flt3 ligand. Blood 2003;102:2513-21.

Della Porta M, Danova M, Rigolin GM, Brugnatelli S, Rovati B, Tronconi $C$, et al. Dendritic cells and vascular endothelial growth factor in colorectal cancer: correlations with clinicobiological findings. Oncology 2005;68:276-84.

Demedts IK, Bracke KR, Maes T, Joos GF, Brusselle GG. Different roles for human lung dendritic cell subsets in pulmonary immune defense mechanisms. Am J Respir Cell Mol Biol 2006;35:387-93.

Donaghy H, Pozniak A, Gazzard B, Qazi N, Gilmour J, Gotch F, et al. Loss of blood CDIlc(+) myeloid and CDIlc(-) plasmocytoid dendritic cells in patients with HIV-1 infection correlates with HIV-1 RNA virus load. Blood 2001;98:2574-76.

Dzionek A, Fuchs A, Schmidt P, Cremer S, Zysk M, Myltenyi S, et al. BDCA-2, BDCA- 3, BDCA-4: three markers for distinct subsets of dendritic cells in human peripheral blood. J Immunol 2000; 165:6037-46.

Ferrari S, Rovati B, Cucca L, Porta C, Alessandrino PE, Bertolini A, et al. Lack of dendritic cell mobilization into the peripheral blood of cancer patients following standard- or high-dose chemotherapy plus granulocyte-colony stimulating factor. Cancer Immunol Immunother 2003;52:359-66.

Ferrari S, Malugani F, Rovati B, Porta C, Riccardi A, Danova M. Flow cytometric analysis of circulating dendritic cell subsets and intracellular cytokine production in advanced breast cancer patients. Oncol Rep 2005; 14:113-20.

Finke JS, Shodell M, Shah K, Siegal FP, Steinman RM. Dendritic cell numbers in the blood of HIV-1 infected patients before and after changes in antiretroviral therapy. J Clin Immunol 2004;24: 647-52.

Grassi FR, Hosmalin A, McIloy D, Calvez V, Debré P, Autran B. CD11Cpositive dendritic cells are depleted in the blood of HIV-infected patients. AIDS 1999;13:759-66.

Grouard G, Rissoan MC, Filgueira L, Durand I, Banchereau J, Liu YJ. The enigmatic plasmocytoid $T$ cells develop into dendritic cells with interleukin (IL)-3 and CD40-ligand. J Exp Med 1997;185:110111.

Hart DN. Dendritic cells : unique leukocyte populations which control the primary immune response. Blood 1997; 90:3245-87.

Henrikson SE, von Andrian UH. Single-cell dynamics of T-cell priming. Current Opinion in Immunology 2007;19:249-58.

Ho CS, Lopez JA, Vuckovic S, Pyke CM, Hockey RL, Hart DN. Surgical and physical stress increases circulating blood dendritic cells counts independently of monocyte counts. Blood 2001;98:1405.

Jung $T$, Schauer $U$, Heusser $C$, Neumann C, Rieger C. Detection of intracellular cytokines by flow cytometry. J Immunol Meth 1993; 159:197-07.

LiuYJ, Kanzler H., Soumelis V, Gilliet M. Dendritic cell lineage, plasticity and cross-regulation. Nat Immunol 2001; 2:585-89(a).

LiuYJ. Dendritic cell subsets and lineages, and their functions in innate and adaptive immunity. Cell 2001;106:259-62(b).

Lukacs-Kornek V, Engel D, Tacke F, Kurts C. The role of chemokines and their receptors in dendritic cell biology. Front Biosci, 2008; 13:2238-52.

MacDonald KP, Munster DJ, Clark GJ, Dzionek A, Schimtz J, Hart DN. Characterization of human blood dendritic cell subsets. Blood 2002;100:4512-20.

Mami NB, Mohty M, Chambost H, Gaugler B, Olive D. Blood dendritic cells in patients with acute lymphoblastic leukaemia. $\mathrm{Br} J$ Haematol 2004;126:77-80.

Narbutt J, Lesiak A, Zak-Prelich M, Wozniacka A, Sysa-Jedrzejoswska A, Tybura M, et al. The distribution of peripheral blood dendritic cells assayed by a new panel of anti-BDCA monoclonal antibodies in healthy representatives of the polish population. Cell Mol Biol Lett 2004; 9:497-09.

Patterson S, Donaghy H, Amjadi P, Gazzard B, Gotch F, Kelleher Peter. Human BDCA-1 positive dendritic cells differenziate into phenotypically distinct immature and mature populations in the absence of exogenous maturation stimuli: differentiation failure in HIV infection. The Journal of Immunology, 2005; 174: 8200-9.

Pichyangkul S, Endy TP, Kalayanarooj S, Nisalak A, Yongvanitchit K, Green $S$, et al. A blunted blood plasmocytoid dendritic cell response 
to an acute systemic viral infection is associated with increased disease severity. J Immunol 2003;171:5571-8.

Pilichowska ME, Fleming MD, Pinkus JL, Pinkus GS. CD4 ${ }^{+} / C^{2} 56$ hematodermic neoplasm (blastic natural killer cell lymphoma): neoplastic cells express the immature dendritic cells marker BDCA-2 and produce interferon. Am J Clin Pathol 2007;128:445-53.

Pinzon-Charry A, Ho CSK, Laherty R, Maxwell T, Walker D, Gardiner RA, et al. A population of HLA-DR+ immature cells accumulates in the blood dendritic cell compartment of patients with different types of cancer. Neoplasia 2005;7: 1112-22.

Rissoan MC, Soumelis V, Kadowaki N, Grouard G, Briere F, de Waal Malefyt $R$, et al. Reciprocal control of $T$ helper cell and dendritic cell differentiation. Science 1999;283:1183-6.

Servet C, Zitvogel L, Hosmalin A. Dendritic cells in innate immune response against HIV. Curr Mol Med 2002;2:739-56.

Shortman K, Liu YJ. Mouse and human dendritic cells subtypes. Nature Rev Immunol 2002;3:151-61.

Shurin MR, Shurin GV, Chatta GS. Aging and the dendritic cell system: Implications for cancer. Critical Rev Oncol/Hematol 2007;64:90105.

Sozzani S, Rusnati M, Riboldi E, Mitola S, Presta M. Dendritic cellendothelial cell cross-talk in angiogenesis. Trends in Immunology, 2007;28:385-92.

Steinmann K, Cohn Z. Identification of a novel cells type in peripheral lymphoid organs of mice. J Exp Med 1973;137:1142-62.

Tacke F, Randolph GJ. Migratory fate and differentation of blood monocyte subsets. Immunobiology 2006; 211: 609-18.

Thurnher M. Lipids in dendritic cell biology: messengers, effectors, and antigens. J Leukoc Biol 2007; 81:154-60.

Tsoumakidou M, Tzanakis N, Papada HA, Koutala H, Siafakas N M. Isolation of myeloid and plasmocytoid dendritic cells from human bronchoalveolar lavage fluid. Immunology Cell Biology 2006;84: 267-73.

Vuckovic S, Gardiner D, Field K, Chapman GV, Khailil D, Gill D et al. Monitoring dendritic cells in clinical practice using a new whole blood single- platform TruCOUNT assay. J Immunol Meth 2004;284:73-87.

Whitby L, Granger V, Storie I, Goodfellow K, Sawle A, Reilly JT et al. Quality control of CD4+ T-lymphocyte enumeration: results from 9 years of the United Kingdom National External Quality Assessment Scheme for Immune Monitoring (1993-2001). Cytometry, 2002;50:102-10.

Wilczynski JR, Radwan M, Kalinka J. The characterization and role of regulatory $T$ cells in immune reactions. Front Biosci 2008;13: 2266-74.

Zaba LC, Fuentes-Duculan J, Steinman RM, Krueger JG, Lowes MA. Normal human dermis contains distinct populations of CDIC+ BDCA-1+ dendritic cells and CD163+ FXIIIA+ macrophages. The Journal of Clinical Investigation 2007;117: 2517-25. 
B. Rovati et al.

52 